
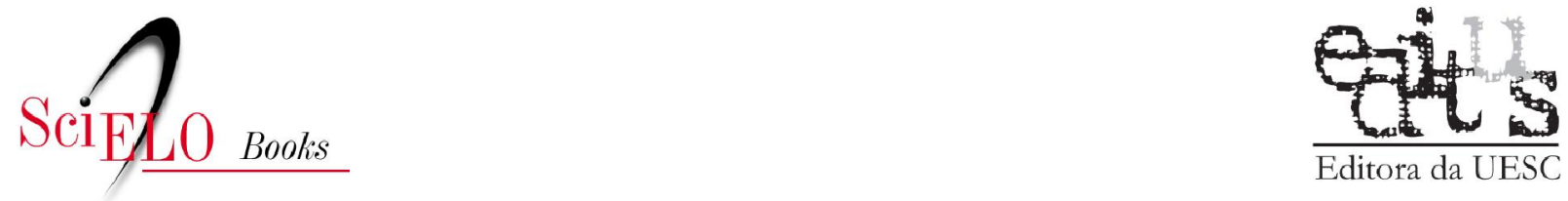

Editora da UESC

\title{
4 - Estado da arte sobre a taxonomia e filogenia de Heteroponerinae
}

\author{
Rodrigo M. Feitosa
}

SciELO Books / SciELO Livros / SciELO Libros

FEITOSA, RM. Estado da arte sobre a taxonomia e filogenia de Heteroponerinae. In: DELABIE, JHC., et al., orgs. As formigas poneromorfas do Brasil [online]. Ilhéus, BA: Editus, 2015, pp. 33-41. ISBN 978-85-7455-441-9. Available from SciELO Books $<$ http://books.scielo.org $>$.

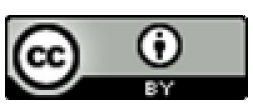

All the contents of this work, except where otherwise noted, is licensed under a Creative Commons Attribution $\underline{4.0 \text { International license. }}$

Todo o conteúdo deste trabalho, exceto quando houver ressalva, é publicado sob a licença Creative Commons Atribição 4.0.

Todo el contenido de esta obra, excepto donde se indique lo contrario, está bajo licencia de la licencia Creative Commons Reconocimento 4.0. 


\title{
Estado da arte sobre a taxonomia e filogenia de Heteroponerinae
}

\author{
Rodrigo M. Feitosa
}

\section{Resumo}

Heteroponerinae compreende três gêneros de formigas: Acanthoponera Mayr, Aulacopone Arnol'di e Heteroponera Mayr. Acanthoponera é exclusivamente Neotropical, enquanto Heteroponera mostra uma distribuição disjunta nas Américas e Oceania. O gênero Aulacopone ocorre apenas na Região Paleártica. A principal característica morfológica exclusiva da subfamília é a presença de uma carena longitudinal mediana, que se estende da margem anterior do clípeo à margem posterior da cabeça.

Acanthoponera é representado por quatro espécies neotropicais. Heteroponera apresenta 19 espécies, seis das quais restritas à Região Australiana e 13 exclusivamente neotropicais. Aulacopone é conhecido por uma única espécie, $A$. relicta, representada por um par de rainhas coletadas por volta da década de 1930 em duas localidades no sudoeste da Ásia (Azerbaijão).

No que se refere às relações filogenéticas internas em Heteroponerinae, tanto a subfamília quanto os gêneros são monofiléticos. Aulacopone aparece em uma posição basal e representa o grupo-irmão do clado formado por Acanthoponera + Heteroponera. No caso do gênero mais especioso e também o único desta subfamília presente em mais de uma região biogeográfica, Heteroponera, as relações internas indicam que as espécies australianas ocupam uma posição basal em comparação às neotropicais. Ainda, as espécies encontradas em regiões andinas da América do Sul parecem estar mais proximamente relacionadas às espécies australianas do que às demais espécies neotropicais.

Observações de campo aliadas a dados de rótulo de espécimes de museus fornecem importantes informações acerca da história natural do grupo. Larvas de Heteroponerinae são predominantemente predadoras, alimentando-se de pequenos artrópodes coletados pelas operárias. Ocasionalmente, adultos podem se alimentar dos líquidos (hemolinfa) resultantes das feridas provocadas no tegumento das presas caçadas por operárias e de exsudatos vegetais no caso de espécies arborícolas. Os membros de Heteroponera têm hábitos predominantemente diurnos e seus ninhos são encontrados no solo, serapilheira e troncos em decomposição, e podem abrigar colônias com algumas dezenas a poucas centenas de indivíduos, sendo que as operárias forrageiam preferencialmente no solo. Espécies de Acanthoponera são noturnas, nidificando e forrageando exclusivamente em árvores.

Com relação à estratégia reprodutiva, algumas espécies de Heteroponera podem apresentar rainhas ergatoides dividindo a função reprodutiva com as rainhas aladas na mesma colônia, enquanto em outras espécies as rainhas aladas foram completamente substituídas por ergatoides. Até onde se sabe, todas as espécies de Acanthoponera possuem rainhas aladas.

FEITOSA, Rodrigo M. Estado da arte sobre a taxonomia e filogenia de Heteroponerinae. In: DELABIE, Jacques H. C. et al. As formigas poneromorfas do Brasil. Ilhéus: Editus, 2015. p. 33-41. 
State of the art of the taxonomy and phylogeny of the subfamily Heteroponerinae - Heteroponerinae comprises three ant genera: Acanthoponera Mayr, Aulacopone Arnol'di and Heteroponera Mayr. Acanthoponera is exclusively Neotropical, while Heteroponera shows a disjoint distribution in the Americas and Australia. Aulacopone is known only from the Paleartic Region.

Acanthoponera is represented by four Neotropical species. Heteroponera possess 19 species, six of which are restricted to the Australian Region and 13 are exclusively Neotropical. Aulacopone is known from a single species, $A$. relicta, represented by two queens collected around the 1930s in two localities in Southeast Asia (Azerbaijan).

The phylogeny of the group demonstrates the monophyly of Heteroponerinae and its genera. The internal relationships indicate a basal position of the paleartic genus Aulacopone, which appears as sister group to the clade Acanthoponera + Heteroponera. Regarding Heteroponera, the most diverse heteroponerine genus, the presence of these lineages in at least two biogeographic regions and the phylogenetic proximity found between Andean and Australian groups reveal an interesting biogeographic scenario.

Field observations allied to label data from museum specimens provide important information about the natural history of the group. Larvae of Heteroponerinae are predominantly predators, and workers are occasionally observed feeding on insect and plant exudates. Nests can support colonies with dozens to a few hundred of individuals. Heteroponera are diurnal and can nest in soil and vegetation, but preferentially forage in the soil, while Acanthoponera is nocturnal and exclusively arboreal.

With respect to the reproductive strategy, some species of Heteroponera may possess ergatoid queens, which can share the reproductive function with dealate queens, while in other species the winged queens are completely replaced by ergatoids. As far as we know, all species of Acanthoponera have winged queens.

\section{Sistemática de Heteroponerinae}

Por mais que os progressos recentes tenham trazido luz ao conhecimento sobre taxonomia e as relações filogenéticas entre as principais linhagens de formigas em um nível abrangente (ver Capítulo 7 deste livro por Fernández; Lattke), muito ainda está por ser compreendido acerca da filogenia interna de boa parte das subfamílias, tribos ou mesmo gêneros. Este é o caso da subfamília Heteroponerinae. Até recentemente, as formigas desta subfamília eram consideradas como pertencentes à tribo Ectatommini que, juntamente com outras tribos, formavam um táxon muito abrangente e heterogêneo, a subfamília Ponerinae (BOLTON, 1994; BOLTON, 1995).

Brown (1958) apresentou a primeira proposta de classificação para Ectatommini. Neste trabalho, uma série extensiva e bem justificada de sinonímias genéricas reduziu um imenso e confuso arranjo de nomes a apenas nove gêneros (Acanthoponera, Aulacopone, Discothyrea, Ectatomma, Gnamptogenys, Heteroponera,
Paraponera, Proceratium e Rhytidoponera). Apesar de não ter realizado uma análise cladística, Brown comparou a morfologia de Ectatommini a de diversos outros grupos de Ponerinae, concluindo que Ectatommini apresentava os gêneros mais basais, sendo Acanthoponera o gênero que reunia o maior número de características ancestrais e do qual se derivava diretamente Heteroponera que, por sua vez, seria mais intimamente relacionado à Aulacopone, Discothyrea, Gnamptogenys, Proceratium e Rhytidoponera, enquanto Ectatomma e Paraponera formariam uma linhagem distinta.

Lattke (1994) foi o primeiro a propor uma análise filogenética para Ectatommini utilizando um conjunto abrangente de caracteres de morfologia externa. Em seu estudo, Lattke demonstrou que Ectatommini era um grupo polifilético e propôs uma nova classificação para os gêneros que o compunha, revalidando as tribos Paraponerini, com Paraponera como único membro e Proceratiini, englobando Discothyrea e Proceratium. Os demais gêneros foram mantidos em Ectatommini, que passou a ser representada por dois clados, sendo um 
deles formado por Acanthoponera e Heteroponera (grupo Heteroponera), enquanto o segundo englobava Ectatomma, Gnamptogenys e Rhytidoponera (grupo Ectatomma). Contudo, Ectatommini permaneceu um grupo polifilético, uma vez que os clados que a compunham não compartilhavam o mesmo ancestral em nenhuma das topologias obtidas por Lattke (Figura 4.1A).
Os resultados de Lattke foram duramente contestados por Keller (2000) que afirmou que a classificação proposta por este autor era cladisticamente inconsistente, já que a tribo Ectatommini era polifilética e suas análises foram baseadas em propostas filogenéticas antigas e irrelevantes para a escolha dos grupos externos. Keller então ampliou a matriz de Lattke com novos táxons e caracteres e

Figura 4.1 - Relações entre os gêneros de Ectatommini com as propostas para a reclassificação da tribo segundo (A) Lattke (1994) e (B) Keller (2000). A barra azul mostra a posição dos gêneros heteroponeríneos incluídos nas análises (Modificado de Feitosa, 2011)
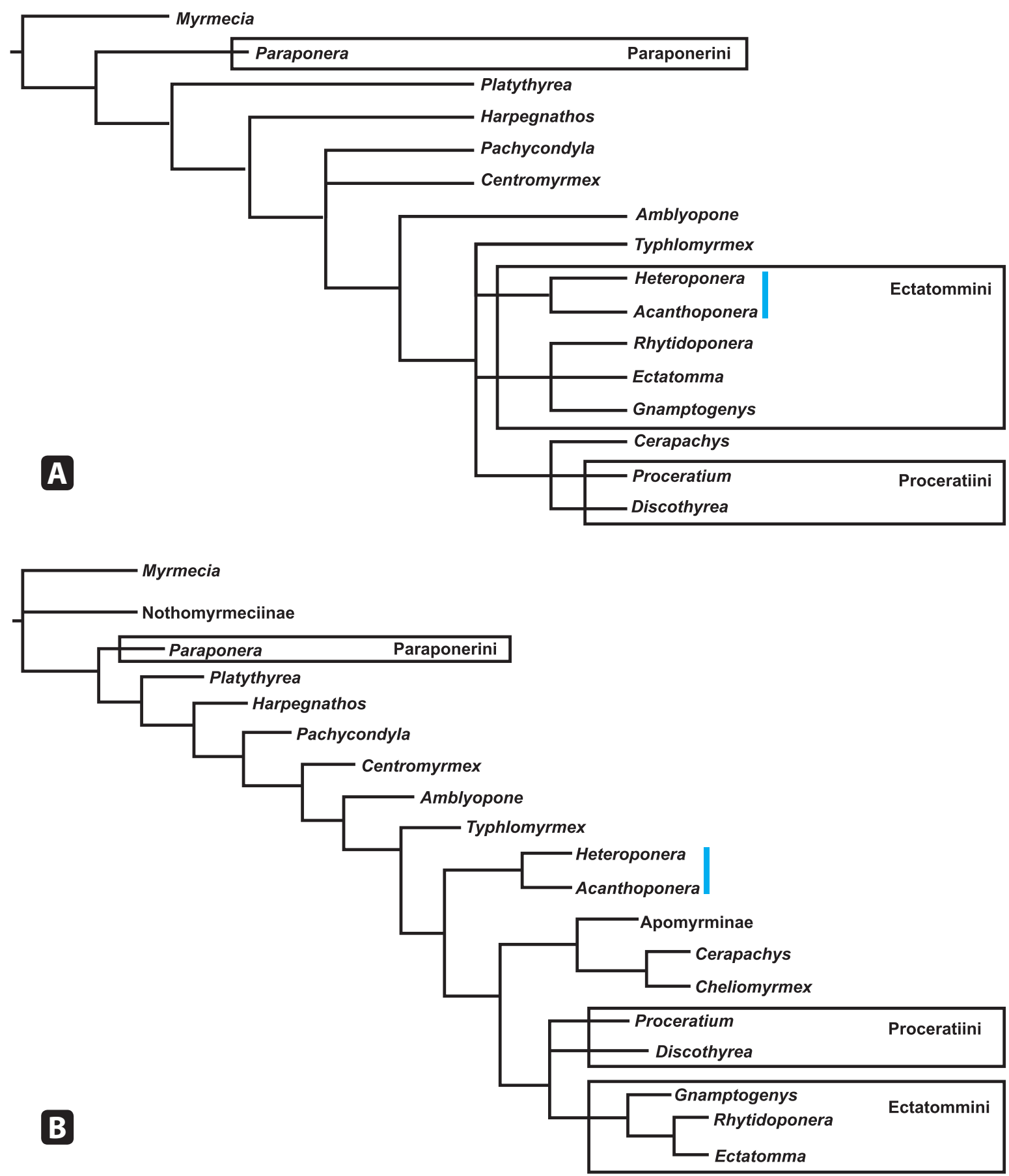
desenvolveu uma nova análise filogenética (Figura 4.1B). Seus resultados confirmaram a distância filogenética entre Paraponera e os demais gêneros e também a monofilia dos grupos Heteroponera e Ectatomma. Contudo, surpreendentemente, o grupo Heteroponera aparece como grupo-irmão de um clado formado por membros de outras subfamílias de formigas (Apomyrminae e Cerapachyinae) e que também inclui o grupo Ectatomma. Estes resultados contrastam drasticamente com o observado em todos os estudos anteriores, perpetuando a instabilidade nas classificações de Ectatommini.

Dadas as evidências de que Ectatommini e mesmo Ponerinae constituíam agrupamentos artificiais, Bolton (2003) desenvolveu um estudo morfológico minucioso e extremamente abrangente e decidiu dividir Ponerinae em seis subfamílias: Amblyoponinae, Ectatomminae, Heteroponerinae, Paraponerinae, Ponerinae e Proceratiinae que se somaram às demais 15 subfamílias existentes. Os gêneros que até então formavam a tribo Ectatommini agora se encontram divididos nas subfamílias Ectatomminae (Ectatomma, Gnamptogenys, Rhytidoponera e Typhlomyrmex), Heteroponerinae (Acanthoponera, Aulacopone e Heteroponera) e Proceratiinae (Discothyrea, Probolomyrmex e Proceratium).

No mesmo trabalho, Bolton (2003) considerou as subfamílias originadas do desmembramento de Ponerinae como membros de um agrupamento informal, o qual chamou de poneromorfas. Este termo teve aceitação por algum tempo e apareceu com frequência na literatura dos anos seguintes ao trabalho de Bolton. No entanto, filogenias recentes utilizando dados moleculares demonstraram que as poneromorfas formam, na verdade, uma assembleia artificial, com um grupo monofilético (o complexo poneroideo), excluindo-se as subfamílias Ectatomminae e Heteroponerinae que juntas formam o complexo ectaheteromorfo e que, ao lado de Formicinae e Myrmicinae, compõem o atual clado formicoide (MOREAU et al., 2006; OUELLETTE et al., 2006; BRADY et al., 2006; RABELING et al., 2008) (Figura 4.2).

Mais recentemente, Keller (2011) publicou uma das análises filogenéticas mais abrangentes já feitas para formigas, utilizando exclusivamente caracteres morfológicos, com ênfase nos grupos incluídos no complexo poneromorfo de Bolton (2003). Apesar das grandes divergências deste trabalho com as propostas recentes que utilizaram dados moleculares, a proposta de Keller comprova a parafilia do complexo poneromorfo e a relação de grupo-irmão entre Ectatomminae e Heteroponerinae (grupo ectaheteromorfo).

Feitosa (2011) desenvolveu uma revisão taxonômica global e a primeira tentativa de analisar as relações filogenéticas internas deHeteroponerinae com base em caracteres morfológicos externos de todos os táxons nela incluídos. A análise filogenética

Figura 4.2 - Sumário das relações filogenéticas entre subfamílias de formigas reconstruídas com base em dados moleculares. Os triângulos representam a diversidade de cada subfamília (Modificado de Keller, 2011).

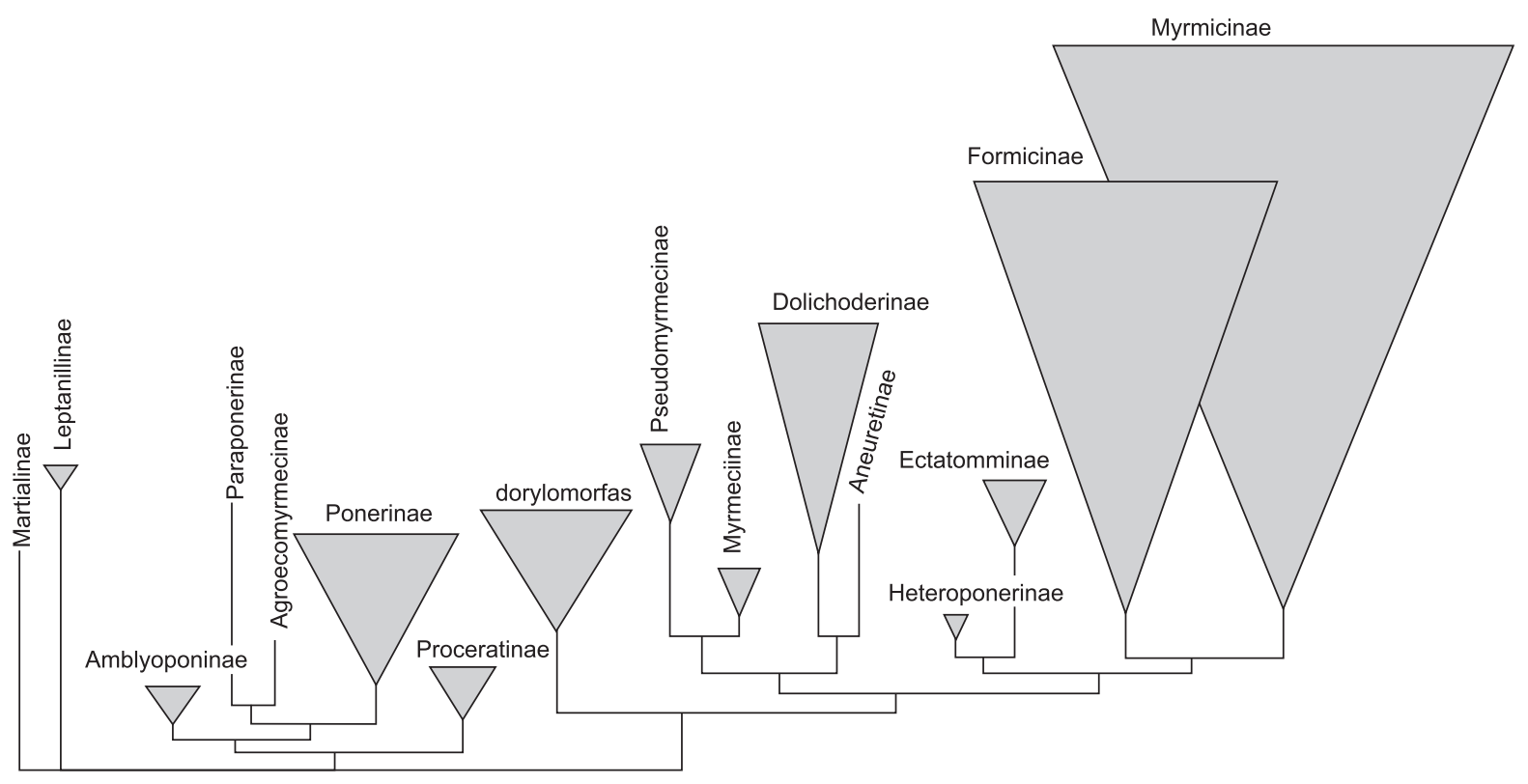


a partir de caracteres morfológicos (Figura 4.3) corroborou a monofilia de Heteroponerinae. Os gêneros Acanthoponera e Heteroponera apareceram como monofiléticos em todas as topologias encontradas. As relações internas apontaram para uma posição basal do gênero paleártico Aulacopone, que figura como grupo-irmão do clado
Acanthoponera + Heteroponera. Este estudo está sendo complementado com a obtenção de dados moleculares para novas inferências sobre a filogenia da subfamília.

A taxonomia alfa de Heteroponerinae sofreu poucas alterações desde o trabalho de Brown (1958). Entre os últimos trabalhos em que foram

Figura 4.3 - Relações filogenéticas internas de Heteroponerinae com base em caracteres morfológicos. Topologia única obtida através da metodologia de pesagem implícita de caracteres $(k=3)$ (Modificado de Feitosa, 2011).

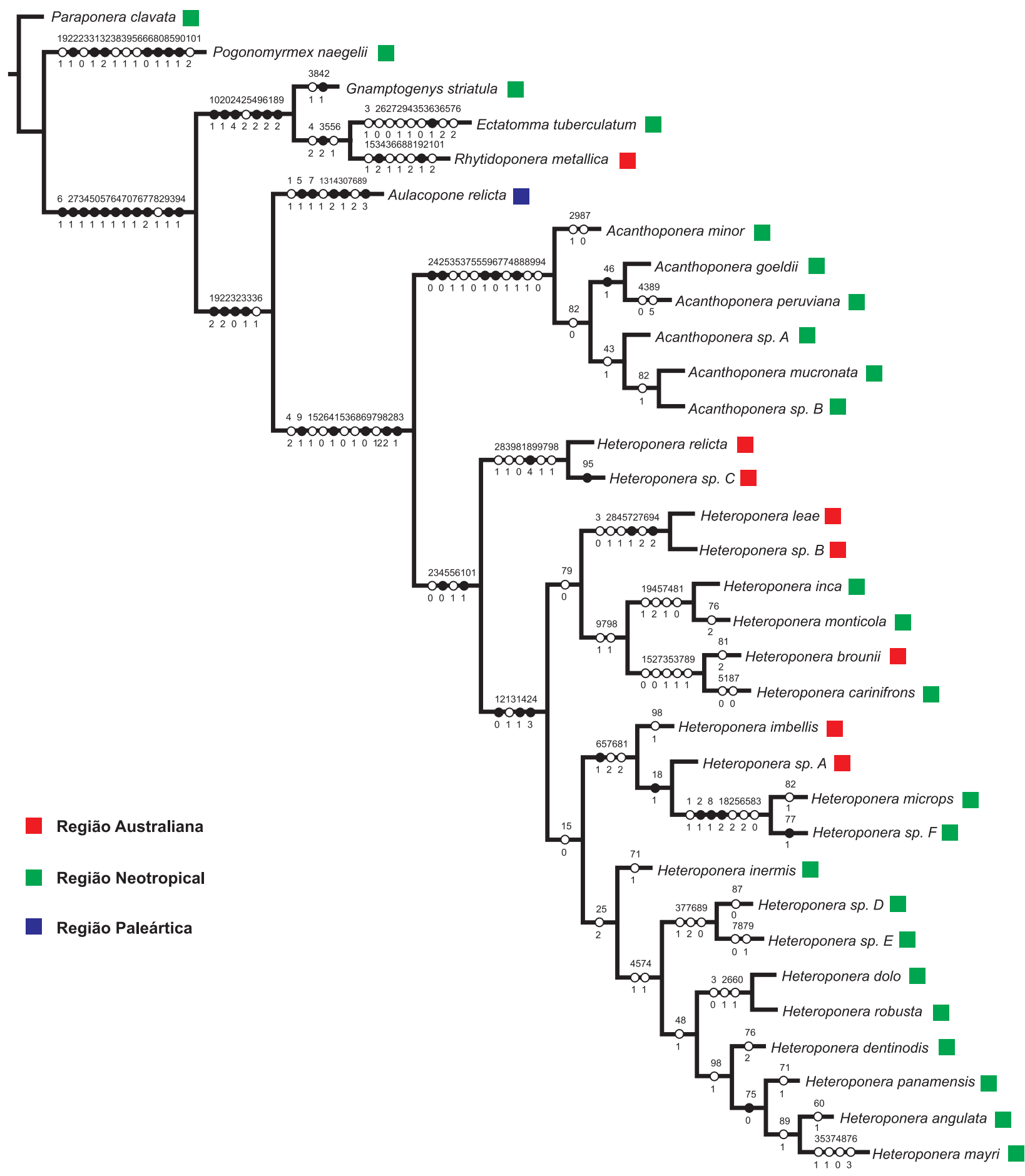


abordados aspectos da taxonomia dos gêneros heteroponeríneos, está o de Kempf (1962), que oferece retoques à classificação das espécies neotropicais de Heteroponera e o artigo de Taylor (2011), com uma revisão das espécies do grupo Heteroponera leae, restritas à Austrália. Desde o trabalho de Brown (1958), nenhuma revisão do gênero Acanthoponera foi realizada, exceto pelo trabalho de Feitosa (2011).

Pelo conceito atual de subfamílias de formigas, Heteroponerinae ainda engloba os três gêneros nela combinados por Bolton (2003): Acanthoponera; Aulacopone (incertae sedis) e Heteroponera, todos reunidos em uma única tribo, Heteroponerini. São formigas de tamanho médio que, apesar de não estarem entre os elementos mais conspícuos da mirmecofauna, podem ser consideradas relativamente comuns dependendo da região. Morfologicamente, caracterizam-se principalmente pela presença de uma carena cefálica longitudinal mediana conspícua, que vai da margem anterior ou posterior do clípeo ao vértice da cabeça; clípeo largamente inserido entre as inserções antenais; antena com 12 artículos; sutura promesonotal presente e flexível e meso e metatíbias com um único esporão cada.

Acanthoponera é representado por quatro espécies neotropicais. Heteroponera apresenta 19 espécies, seis das quais restritas à Região Australiana e 13 exclusivamente neotropicais. Aulacopone é conhecido por uma única espécie, $A$. relicta, representada por um par de rainhas coletadas por volta da década de 1930 em duas localidades no sudoeste da Ásia (Azerbaijão), das quais uma (o holótipo) está perdida.

\section{Representantes de Heteroponerinae no Brasil}

\section{Acanthoponera Mayr, 1862}

Acanthoponera compreende até então quatro espécies (A. goeldii, A. minor, A. mucronata e A. peruviana) caracterizadas principalmente pela presença de escrobos antenais profundos, olhos muito desenvolvidos, espinhos propodeais longos e ápice do pecíolo terminando em um espinho muito desenvolvido (Figura 4.4).

Este é o único gênero de Heteroponerinae restrito exclusivamente à Região Neotropical, ocorrendo do sul do México ao norte da Argentina, preferencialmente em áreas de maior altitude. No Brasil ocorrem três espécies, A. goeldii Forel, A. mucronata (Roger) e A. peruviana Brown. Acanthoponera goeldii está restrita às áreas de Floresta Atlântica do Sul e Sudeste do Brasil, enquanto A. peruviana só é encontrada na Região Amazônica. Já $A$. mucronata possui a maior distribuição entre as espécies do gênero e pode ser encontrada em todas as regiões do país.

A principal referência para a identificação das espécies deste gênero segue sendo o trabalho de Brown (1958) que se baseia principalmente no grau de desenvolvimento dos espinhos propodeais e na profundidade da constrição entre o primeiro e o segundo segmentos gastrais para delimitar as espécies. Estas características têm grande importância taxonômica, mas o material acumulado recentemente em coleções permitiu a observação de novas estruturas morfológicas diagnósticas para espécies e o reconhecimento de pelo menos duas

Figura 4.4 - Acanthoponera mucronata, operária em (a) vista frontal e (b) vista lateral. Foto: Ricardo Kawada.

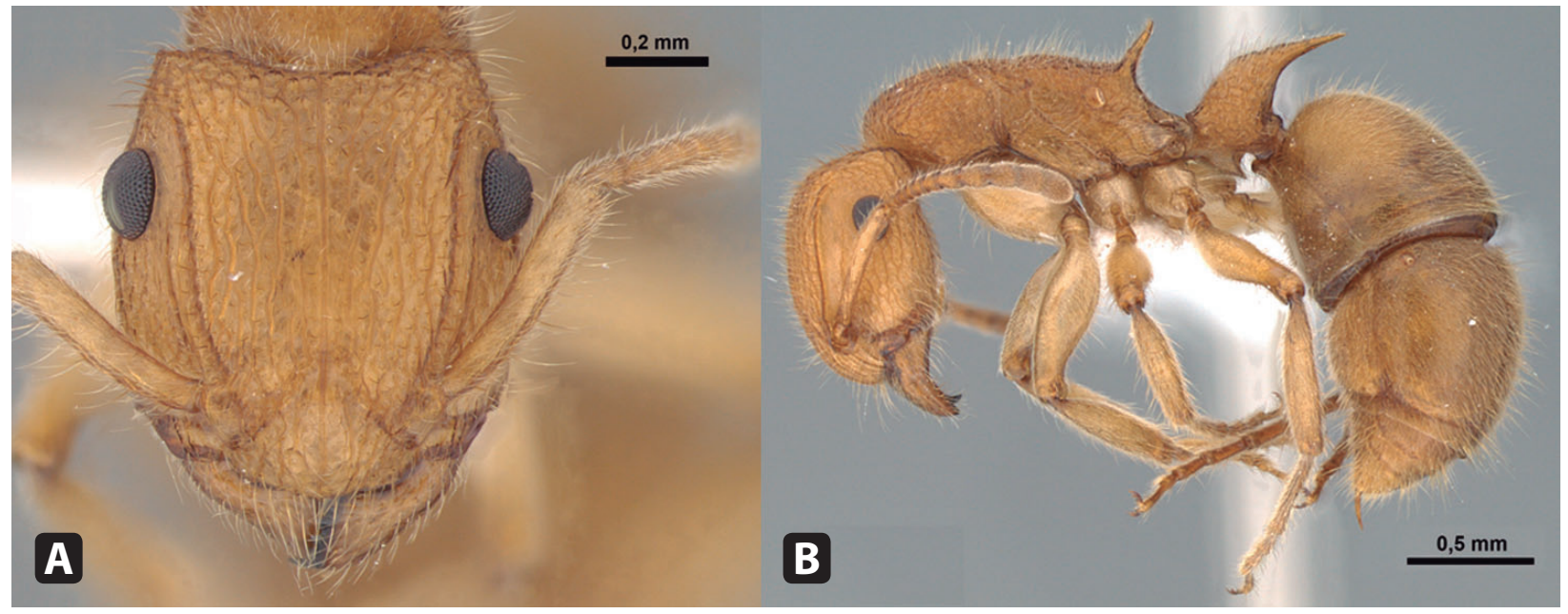


novas espécies para o gênero, uma delas aparentemente endêmica do Sul do Brasil e Uruguai (FEITOSA, 2011).

Operárias de Acanthoponera são formigas robustas que possuem hábitos noturnos e nidificam exclusivamente na vegetação, tanto em florestas úmidas quanto em bosques isolados. De fato, exemplares têm sido coletados em locais com grande atividade antrópica como regiões centrais das cidades de São Paulo e Curitiba, por exemplo (R. FEITOSA, obs. pess.). Por mais singular que possa parecer, a principal técnica de coleta para as formigas deste gênero é a armadilha Malaise de interceptação de voo de insetos. Sendo exclusivamente arborícolas e muito territorialistas, operárias tendem a investigar armadilhas Malaise logo após sua instalação em áreas de floresta e assim acabam capturadas pelos recipientes coletores.

Em seu ambiente natural, as operárias vagam solitariamente por entre galhos e folhas à procura de pequenos artrópodes protegidas pela escuridão noturna. Ao menor sinal de perturbação ou mesmo sob o feixe de luz de uma lanterna, as operárias imediatamente ficam imóveis, dificultando sua localização. Não há relatos sobre estratégias reprodutivas ou estrutura de ninhos.

\section{Heteroponera Mayr, 1887}

Heteroponera é o gênero-tipo e o que abriga o maior número de espécies na subfamília. Treze espécies são conhecidas para a Região Neotropical. As espécies deste gênero podem ser imediatamente diferenciadas do gênero próximo
Acanthoponera pela ausência de um lobo basal nas garras tarsais e pelo menor desenvolvimento dos espinhos propodeais e da projeção posterior do pecíolo (Figura 4.5).

$\mathrm{Na}$ Região Neotropical Heteroponera ocorre da América Central ao centro-sul do Chile, apresentando maior riqueza nas florestas montanhosas da Colômbia e da Mata Atlântica brasileira. De fato, o Brasil abriga oito das 13 espécies descritas para o gênero: $H$. angulata Borgmeier, $H$. dentinodis (Mayr), H. dolo (Roger), H. flava Kempf, $H$. inermis (Emery), H. mayri Kempf, H. microps Borgmeier, e H. robusta Kempf.

A referência até então utilizada para a identificação de espécies de Heteroponera no Brasil é a revisão de Kempf (1962), que ainda é de bastante ajuda na nomeação das espécies que ocorrem nesta região. Contudo, o acúmulo de exemplares deste gênero em coleções entomológicas desde o trabalho de Kempf permitiu um estudo mais abrangente sobre os limites específicos dos membros de Heteroponera que irá resultar em alterações na atual classificação do gênero, incluindo algumas sinonímias e descrições de espécies novas (FEITOSA, 2011).

Os ninhos de Heteroponera são normalmente encontrados em áreas de floresta úmida, no interior de troncos em decomposição caídos, entre folhas nas camadas mais profundas da serapilheira e eventualmente na vegetação, onde ocupam cavidades no interior de galhos vivos ou mortos ou nidificam entre as raízes de epífitas. As colônias são relativamente pequenas, em geral com menos de 200 operárias. Quando perturbadas, as operárias

Figura 4.5 - Heteroponera mayri, operária em (a) vista frontal e (b) vista lateral. Foto: Ricardo Kawada.

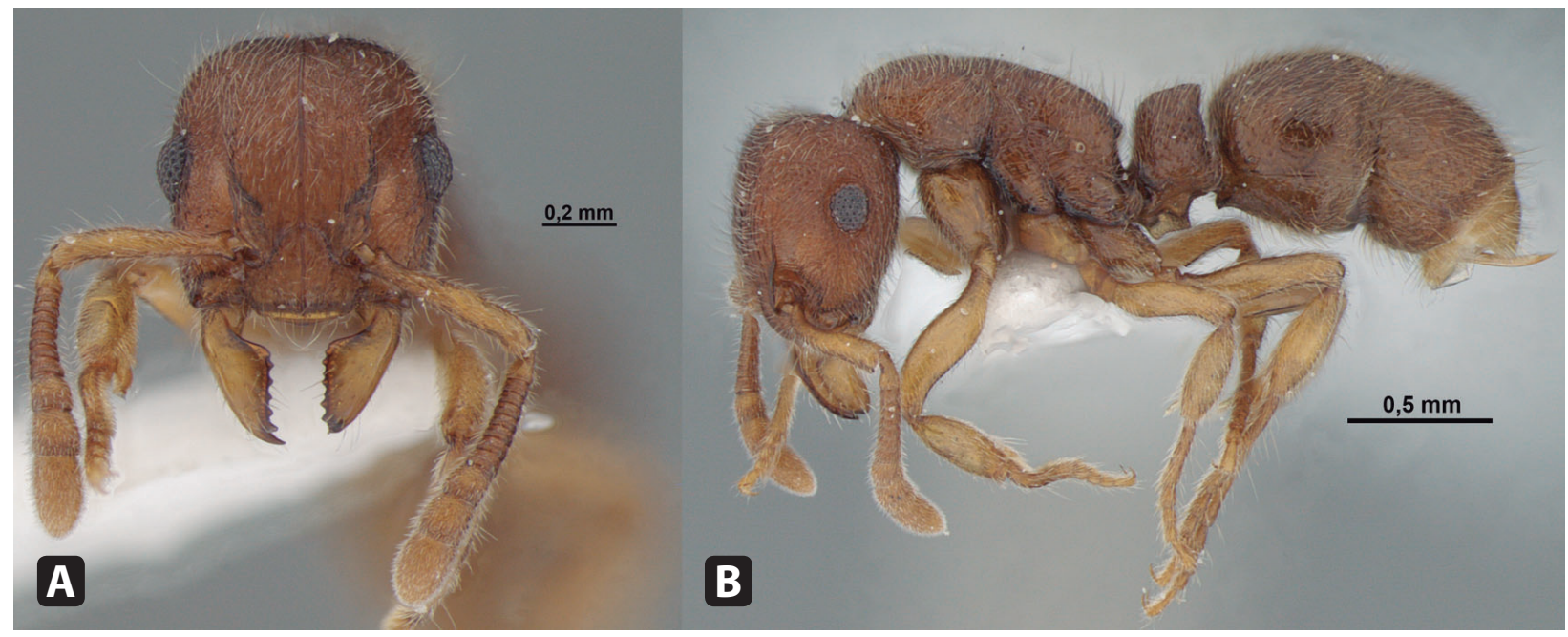


tendem a cercar a prole e posicionar o gáster para cima sem, no entanto, mostrarem-se agressivas, permanecendo imóveis ou fingindo-se de mortas (tanatose) (LATTKE, 2003; BORGES et al., 2004; LONGINO, 2005).

A ergatoginia é um fenômeno comumente observado em Heteroponera. Nas espécies em que esta estratégia reprodutiva foi registrada, além das gines ergatoides, normalmente também estão presentes gines aladas. A fundação de novas colônias se dá, muito provavelmente, por fissão, como demonstram alguns experimentos conduzidos em laboratório (FRANÇOSO, 1995; FEITOSA, 2011).

Em laboratório, espécies de Heteroponera aceitam preferencialmente larvas e adultos de Tenebrio mollitor (Coleoptera: Tenebrionidae), larvas de Alphitobius sp. (Coleoptera: Tenebrionidae), adultos de Falsonia candida (Collembola) e larvas de Drosophila sp. (Diptera) (FRANÇOSO, 1995). Na natureza, diversas operárias foram observadas transportando pequenos artrópodes (inteiros ou em pedaços) para a colônia (FEITOSA, 2011).

\section{Perspectivas}

Na revisão taxonômica de Feitosa (2011), após o exame de 33 dos 36 espécimes-tipo designados para as espécies e subespécies de Heteroponerinae, seis espécies foram reconhecidas para o gênero Acanthoponera, duas ainda não descritas. Heteroponera apresenta 21 espécies (seis delas descritas pela primeira vez), sete das quais restritas à Região Australiana (três novas) e 14 exclusivamente neotropicais (três novas). Duas sinonímias foram propostas para espécies neotropicais. Indivíduos de castas e sexos ainda não registrados (incluindo machos, gines e operárias) foram descritos pela primeira vez para diferentes espécies de Heteroponerinae. Novos registros de localidades expandiram significativamente a distribuição conhecida para a maior parte das espécies.

$\mathrm{O}$ estudo de Feitosa lançou bases seguras para o reconhecimento e delimitação de grande parte das espécies da subfamília e representou o primeiro passo na tentativa de se compreender as relações filogenéticas internas em Heteroponerinae. Neste mesmo estudo fica evidente o quão tênues são os limites morfológicos entre Heteroponerinae e seu grupo-irmão, a subfamília Ectatomminae.
Neste sentido, em nome da estabilidade taxonômica em Formicidae, justifica-se a sinonímia de Heteroponerinae sob Ectatomminae, uma vez que esta última detém a prioridade taxonômica por englobar o gênero mais antigo do agrupamento (Ectatomma Smith, 1858). Este ato nomenclatural, assim como as novas espécies reconhecidas neste estudo, serão formalmente propostos pelo presente autor em um manuscrito a ser publicado oportunamente.

\section{Referências}

BOLTON, B. Identification Guide to the Ant Genera of the World. Harvard University Press. Cambridge, Massachusetts, p. 222, 1994.

BOLTON, B. A New General Catalogue of the Ants of the Word. Harvard University Press. Cambridge, Massachusetts. 1995. 504 p.

BOLTON, B. Synopsis and classification of Formicidae. Memoirs of the American Entomological Institute, v. 71, p. 1-370, 2003.

BORGES, D. S.; DELABIE, J. H. C.; MARIANO, C. S. F.; POMPOLO, S. G. Notes écologiques et étude cytogénétique de la Fourmi néotropicale Heteroponera dolo (Roger, 1981) (Hymenoptera, Formicidae, Heteroponerinae). Bulletin de la Société Entomologique de France, v. 109, p. 257-261, 2004.

BRADY, S. G.; SCHULTZ, T. R.; FISHER, B. L.; WARD, P. S. Evaluating alternative hypotheses for the early evolution and diversification of ants. Proceedings of the National Academy of Sciences of the United States of America, v. 13(48), p. 18172-18177, 2006.

BROWN, W. L., Jr. Contributions toward a reclassification of the Formicidae. II. Tribe Ectatommini (Hymenoptera). Bulletin of the Museum of Comparative Zoology of Harvard College, v. 118, p. 175-362, 1958.

\section{FEITOSA, R. S. M. Revisão Taxonômica e Análise Filogenética de Heteroponerinae (Hymenoptera: Formicidae). Tese de Doutorado apresentada ao Programa de Pós-Graduação em Entomologia da Faculdade de Filosofia Ciências e Letras da Universidade de São Paulo. Ribeirão Preto. São Paulo, Brasil. 2011. XIII + 297 p.}

\section{FRANÇOSO, M. F. L. Biologia e Taxonomia de Heteroponera Mayr, 1887 Neotropicais}

(Hymenoptera: Formicidae). Dissertação de mestrado apresentada ao Instituto de Biociências da Universidade de São Paulo. São Paulo, Brasil. 1995. 125 p. 
KELLER, R. A. Cladistics of the tribe Ectatommini (Hymenoptera: Formicidae): a reappraisal. Insect Systematics; Evolution. 2000. v. 31, p. 59-69.

KELLER, R. A. A phylogenetic analysis of ant morphology (Hymenoptera: Formicidae) with special reference to the poneromorph subfamilies. Bulletin of the American Museum of Natural History, v. 355, p. 1-90, 2011.

KEMPF, W. W. Retoques à classificação das formigas do gênero Heteroponera Mayr (Hym., Formicidae). Papéis Avulsos do Departamento de Zoologia, v. 15, p. 29 47, 1962.

LATTKE, J. E. Phylogenetic relationships and classification of ectatommine ants (Hymenoptera: Formicidae). Entomologica Scandinavica, v. 25, p. 105-119, 1994.

LATTKE, J. E. Subfamilia Ponerinae. In: Fernández, F. (Org.). Introducción a las Hormigas de la Región Neotropical. Instituto de Investigación de Recursos Biológicos Alexander von Humbolt. Bogotá. 2003. p. 261-276.

LONGINO, J. T. 2005. Ants of Costa Rica. Publicação eletrônica. Disponível em: http://www.evergreen.edu/ ants/AntsofCostaRica.html. (Acessado em 20 de Julho de 2011).
MAYR, G. L. Myrmekologische Studien.

Verhandlungen der Zoologisch-Botanischen

Gesellschaft in Wien, v. 12, p. 649-776, 1862.

MAYR, G. L. Südamerikanische Formiciden. Verhandlungen der Zoologisch-Botanischen

Gesellschaft in Wien, v. 37, p. 511-632, 1887.

MOREAU, C. S.; BELL, C. D.; VILA, R.; ARCHIBALD, B.; PIERCE, N. E. Phylogeny of the ants: diversification in the age of angiosperms. Science, v. 312, p. 100-104, 2006.

OUELLETTE, G. D.; FISHER, B. L.; GIRMAN, D. J. Molecular systematics of basal subfamilies of ants using 28S rRNA (Hymenoptera: Formicidae). Molecular Phylogenetics and Evolution, v. 40, p. 359369, 2006.

RABELING, C.; BROWN J. M.; VERHAAGH, M. Newly discovered sister lineage sheds light on early ant evolution. Proceedings of the National Academy of Science, v. 105 (39), p. 14913-14917, 2008.

TAYLOR, R. W. Australasian ants of the subfamily Heteroponerinae (Hymenoptera: Formicidae): (1) General introduction and review of the Heteroponera leae (WHEELER, 1923) species group, with descriptions of two new species. Myrmecological News, v. 15, p. 117-123, 2011. 